\title{
LA SEGURIDAD MARÍTIMA EN LA ESTRATEGIA DE SEGURIDAD NACIONAL 2013
}

\author{
Gracia Abad ${ }^{1}$ \\ Universidad de Nebrija / UNISCI
}

\begin{abstract}
Resumen:
La Estrategia de Seguridad Nacional no parece conceder a la seguridad marítima la importancia que debería si se tiene en cuenta tanto la posición geográfica de España como la relevancia que tiene el mar en los intercambios comerciales de nuestro país. En esa misma línea cabe considerar que los planteamientos de la actual Estrategia de Seguridad Nacional son insuficientes tanto en lo que hace a los desafíos identificados en relación con la seguridad marítima, como en lo que respecta a las líneas de acción planteadas como respuesta a tales desafíos. Por otra parte, cabe considerar que, si bien la Subestrategia de Seguridad Marítima Nacional colma en parte algunas de las lagunas dejadas por la Estrategia de Seguridad Nacional en este ámbito, no lo hace en el caso de otras.
\end{abstract}

Palabras clave: Estrategia, seguridad, nacional, marítima, piratería, amenazas, riesgos, energía, gas, petróleo, comercio, migración.

Title in English:"Sea Security in the National Security Strategy 2013"

\begin{abstract}
:
The National Security Strategy does not seem to give due relevance to sea security, bearing in mind Spain's reliance on the sea as derived from its geographical position and the importance of commercial exchanges. Following the same reasoning, we must bear in mind that the document's approach is unsatisfactory both regarding the challenges singled out in relation to our sea security and the responses offered to such challenges. In addition, it must be considered that, even if the National Substrategy of Sea Security fills the gap left in the National Security Strategy, it does it only partially and the results are not fully satisfactory.
\end{abstract}

Keywords: Strategy, Security, National, Sea, Piracy, Threat, Risks, Energy, Gas, Oil, Trade, Migration.

Copyright (C) UNISCI, 2014.

Las opiniones expresadas en estos artículos son propias de sus autores, y no reflejan necesariamente la opinión de UNISCI. The views expressed in these articles are those of the authors, and do not necessarily reflect the views of UNISCI.

\footnotetext{
${ }^{1}$ La Dr. Gracia Abad es profesora de Relaciones Internacionales en la Universidad Nebrija en Madrid, investigadora senior en UNISCI y es miembro del Foro Hispano-Argelino.

E-mail: graciaabad@yahoo.es. 


\section{Introducción}

A la luz de lo expuesto en el Capítulo 1 (p 6) cabría pensar que la prioridad que se otorga a la seguridad marítima no es demasiado alta pues cuando se enumeran nuevos riesgos y amenazas que, junto a los tradicionales, pueden suponer un desafío para la seguridad no se plantean ningún aspecto relacionado con la seguridad marítima cuando se podrían haber mencionado cuestiones como la piratería o, incluso, el medio ambiente.

Tal falta de atención a la seguridad marítima es cuando menos cuestionable si se tiene en cuenta la posición geográfica de España, la importancia de su flota pesquera o el hecho de que algunas de sus principales fuentes de vulnerabilidad estén relacionadas con fenómenos de radicalismo y terrorismo, suministros energéticos, cuestiones migratorias, conflictos territoriales, ausencia de recursos naturales...

Por otra parte, esa falta de atención española contrasta con la creciente importancia que para muchos Estados parece estar adquiriendo la dimensión marítima. En este sentido, parecería que muchos de ellos son más conscientes de que como acertadamente señala el Real Instituto Elcano en un documento de trabajo publicado en octubre de 2013 "el dominio marítimo vuelve a proporcionar flexibilidad estratégica" ${ }^{3}$.

Así, por ejemplo, a diferencia de lo que ocurre en el caso español, por poner un ejemplo, el libro blanco francés si se plantea la importancia del ámbito marítimo para Francia y la presencia de ésta en los océanos ${ }^{4}$.

Con todo sí queda recogida la seguridad marítima en el Capítulo 2 cuando se hace una revisión de la "Seguridad de España en el Mundo", aunque sólo en relación con el caso de África (p 17).

Por otra parte, es justo decir que la seguridad marítima, como otros ámbitos de la seguridad, es objeto de una subestrategia -Estrategia de Seguridad Marítima Nacional- donde sí se abordan con más detalle algunas de estas cuestiones. En concreto, se resalta la dimensión marítima de España y la importancia de la pesca, el transporte marítimo y la biodiversidad marina para su prosperidad y bienestar ${ }^{5}$

En este sentido, en la Estrategia de Seguridad Marítima sí se hace referencia a todo el abanico de riesgos y amenazas que pueden afectar a España, pero mediante un uso confuso de los conceptos donde se habla de "riesgos y amenazas deliberadas" y "riesgos y amenazas derivados de la peligrosidad intrínseca a cualquier actividad realizada en el medio marino", cuando habría sido más claro -y también más correcto- referirse sencillamente a los primeros como amenazas y llamar riesgos a los segundos ${ }^{6}$.

\footnotetext{
${ }^{2}$ Informe Elcano: "Hacia una renovación de la política exterior española: elementos para conectar mejor el proyecto colectivo de país con el mundo globalizado", versión del 10 de octubre de 2013, pp. 16 y ss.

${ }^{3}$ Arteaga, Felix: "Defensa que viene. Criterios para la reestructuración de la Defensa en España", Real Instituto Elcano, Elcano Policy Paper (Octubre de 2013), p. 5.

4 "Livre Blanc Défense et Sécurité National 2013", Direction de L’information Légale et Administrative, París (2013), pp. 13.

5 "Estrategia de Seguridad Marítima Nacional" (2013), p. 1.

${ }^{6}$ Íbid., pp. 3 y 19.
} 


\section{La seguridad marítima en relación con los riesgos $\mathrm{v}$ amenazas a que España puede hacer frente}

Como decíamos, en el capítulo 2, al repasar la Seguridad de España en el Mundo, sí se vincula, acertadamente, la (in)seguridad marítima con un área previamente identificada como especialmente importante para España -el Golfo de Guinea- (p 18) y se señala el impacto tremendamente negativo que podría tener en términos de abastecimiento energético y flujos comerciales.

Tal consideración parece acertada si tenemos en cuenta la inestabilidad de una región con la que, el comercio español de doble vía ha crecido de forma muy visible en los últimos años, especialmente en lo que respecta a las importaciones de gas y petróleo ${ }^{7}$, pero que, como decimos, se trata de una región marcada por una notable debilidad institucional y donde, a los problemas de islamismo y narcotráfico, hay que unir las divisiones étnicas y lingüísticas existentes en la mayoría de los Estados ${ }^{8}$.

No obstante, cabría cuando menos cuestionar que sea el Golfo de Guinea la única área donde un posible déficit de seguridad marítima pueda suponer un desafío para los intereses españoles o, dicho de otro modo, la única zona que es necesario proteger.

Y es que no hay que olvidar que la reducción de la incidencia de la piratería en la costa oriental africana debe mucho a los esfuerzos para proteger esa zona y, en concreto, a la operación Atalanta desarrollada por la Unión Europea y, en su marco, por España desde 2008 y que previsiblemente continuará hasta $2016^{9}$, a la operación Ocean Shield puesta en marcha por la OTAN también en 2008, pero cuya continuidad más allá del año en curso no está en cambio confirmada ${ }^{10}$.

\footnotetext{
${ }^{7}$ Escribano, Gonzalo y Arteaga, Félix: “Seguridad y recursos en el Golfo de Guinea: algunas implicaciones para España", Real Instituto Elcano, ARI 28/2013 (16 de julio de 2013), en http://www.realinstitutoelcano.org/wps/portal/rielcano/contenido?WCM_GLOBAL_CONTEXT=/elcano/elcano _es/programas/energiacambioclimatico/publicaciones/ari28-2013-escribano-arteaga-seguridad-recursos-golfode-guinea-spain.

${ }^{8}$ Canales, Pedro: "Golfo de Guinea: Un nuevo foco de inestabilidad", en Revista Española de Defensa, (Febrero 2014), pp. 47 y ss.

${ }^{9}$ Shelala, Robert: "M. Maritime Security in the Middle East and North África: A Strategic Assessment", CSIS, en http://csis.org/publication/maritime-security-middle-east-and-north-africa-strategic-assessment, pp. 51 y ss.

${ }^{10}$ Ibid., p. 52.
} 


\section{ÁREA PROTEGIDA POR LA OPERACIÓN ATALANTA}

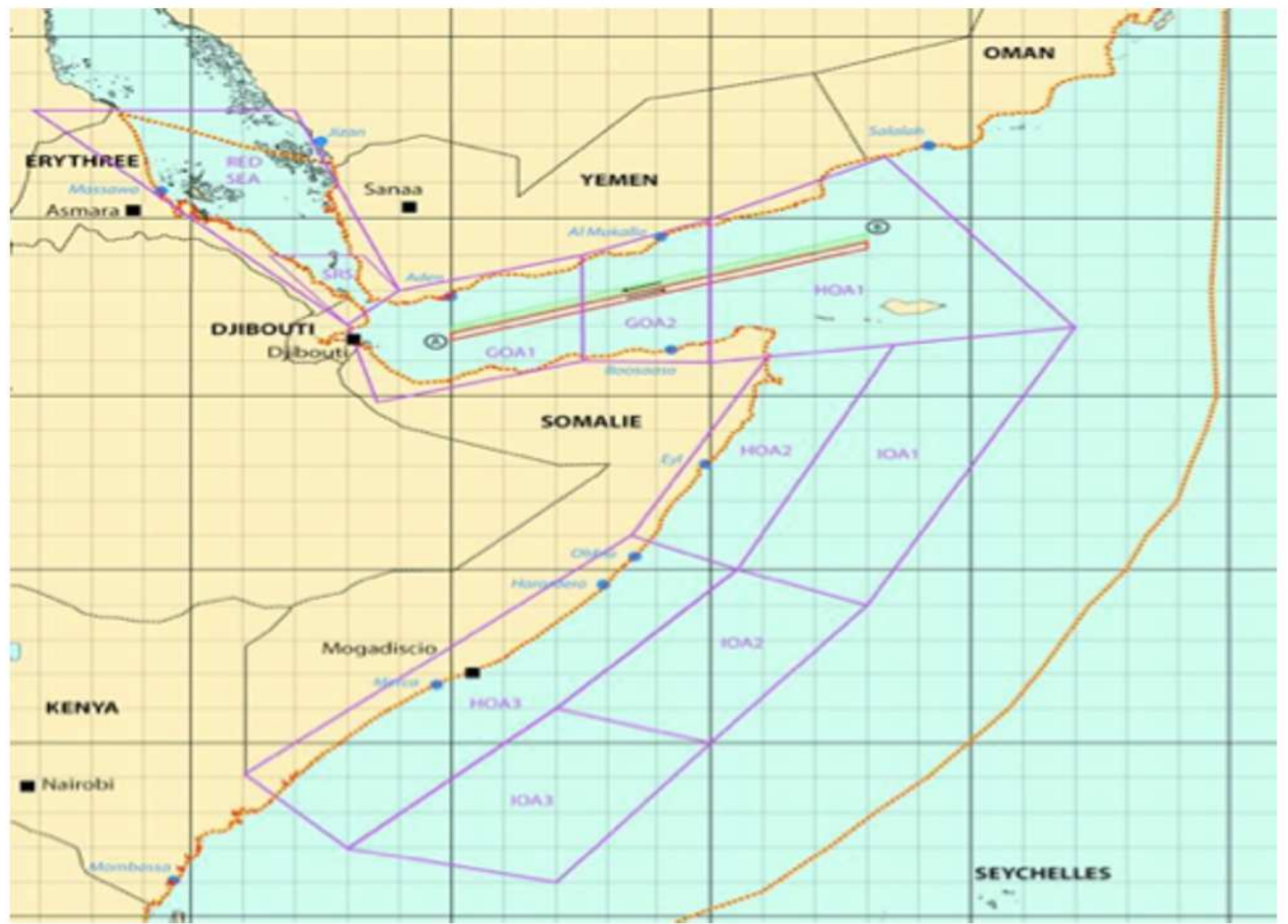

Fuente:http://www.industriaspesqueras.com/noticias/informes/753/zona_de_seguridad_para_cubrir_a_ los_pesqueros.html 


\section{ÁREA AFECTADA POR LA PIRATERÍA SOMALí}

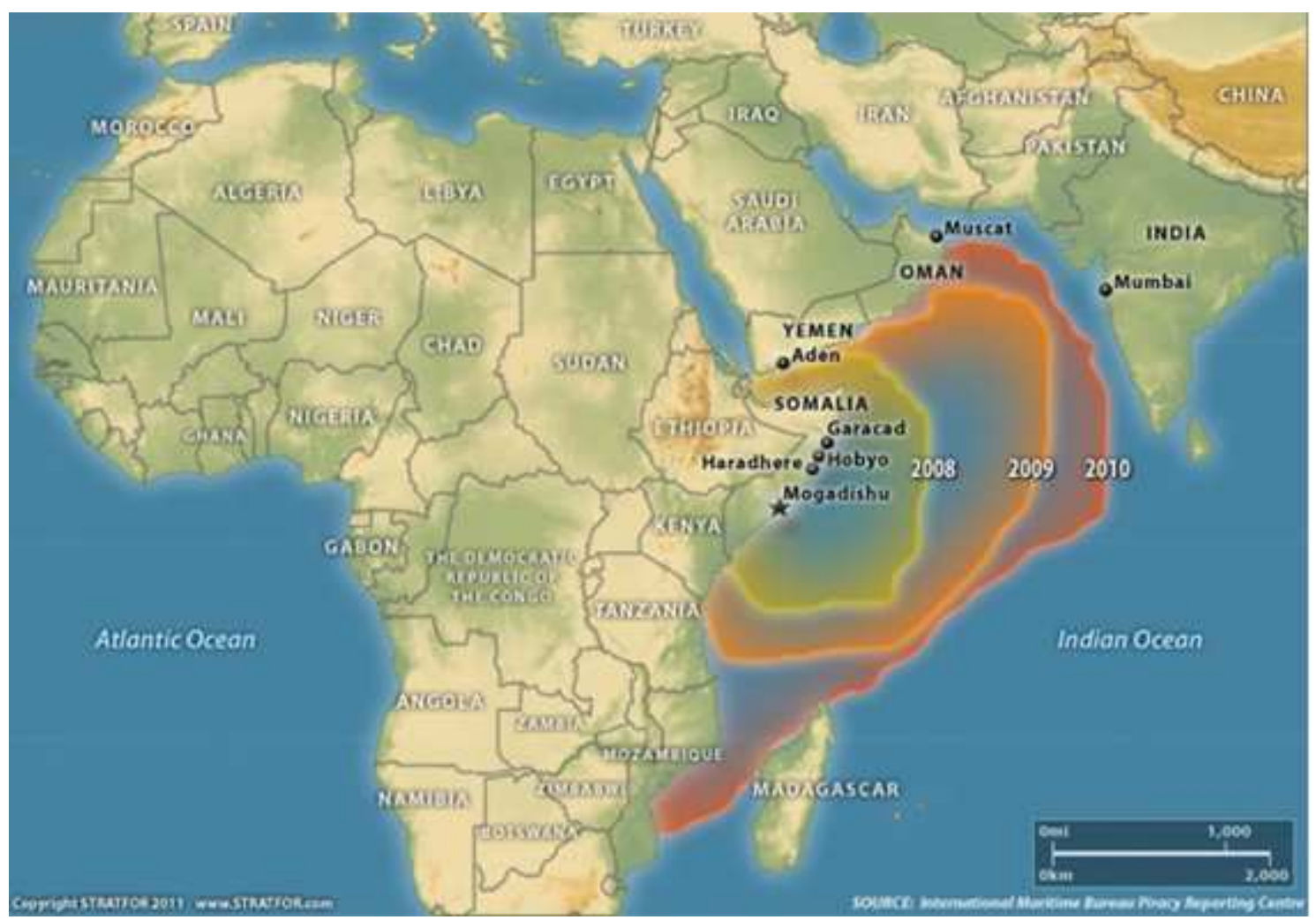

Fuente: http://www.seanews.com.tr/article/PIRACY/71276/Somalia-Piracy-ICS/

El documento pasa así igualmente por alto la importancia que otras zonas, como el Océano

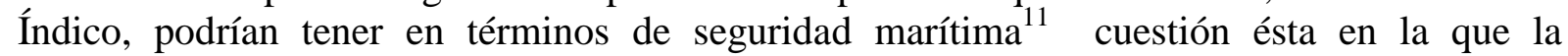
Estrategia de Seguridad Marítima Nacional no difiere sustancialmente de la Estrategia de Seguridad Nacional. Pues bien, la vía del Océano Índico es sin embargo fundamental para el comercio internacional y las comunicaciones entre Europa y Asia.

Al propio tiempo, parece darse por supuesto que sería acertado reproducir en el Golfo de Guinea la estrategia seguida en el Océano Índico y que ha conducido a la reducción piratería en esta área; un planteamiento que pasa por alto las sustanciales diferencias existentes entre las dos áreas, ya que, por ejemplo, mientras el objetivo de los "piratas" en Somalia era el secuestro de pesqueros y la reclamación de rescates, en el Golfo de Guinea el objetivo es el robo de mercancías - petróleo en particular- para su venta ${ }^{12}$.

Por otra parte, sí se establece la relación entre la vulnerabilidad del espacio marítimo y cuestiones como el tráfico de drogas, la migración ilegal o la piratería, como no podía ser de otro modo, en el Capítulo 3, cuando se mencionan los riesgos y amenazas para la seguridad nacional (p 36).

\footnotetext{
${ }^{11}$ Shelala, op. cit., pp. 4 y 39.

12 Pflüger, Juan E.: "La UE copiará la 'Operación Atalanta' en el Occidente de África", La Gaceta, 14 de mayo de 2014, en

http://www.gaceta.es/noticias/ue-copiara-operacion-atalanta-occidente-africa-14052014-1928.
} 
Con todo, el documento no analiza con el detalle que sería deseable la relación entre la seguridad marítima y alguna de esas cuestiones. En particular, sería deseable una reflexión más cuidadosa acerca de la interrelación entre seguridad marítima e inmigración ilegal. Al respecto, el documento no tiene en cuenta ni siquiera las políticas y compromisos asumidos por España en otros ámbitos ya que, por ejemplo, pasa por alto el hecho de que España se ha comprometido en el marco de Naciones Unidas al rescate marítimo de todas aquellas embarcaciones que naufragaran en el área que se extiende al sur de las Islas Canarias y frente a la costa de Marruecos, tal y como muestran los mapas siguientes:

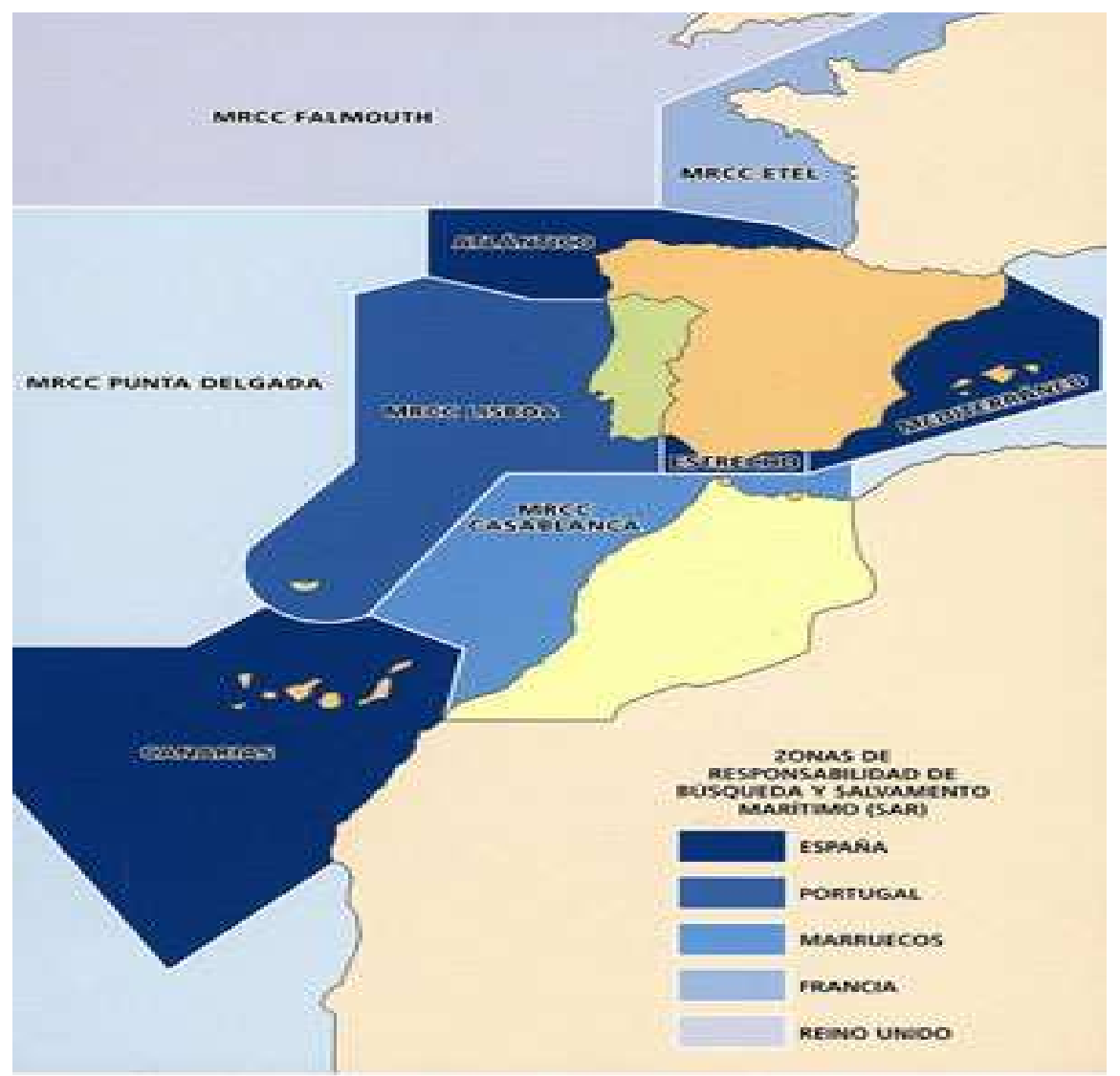

Fuente: http://www.saharalibre.es/modules.php?name=News\&file=print\&sid=4491 


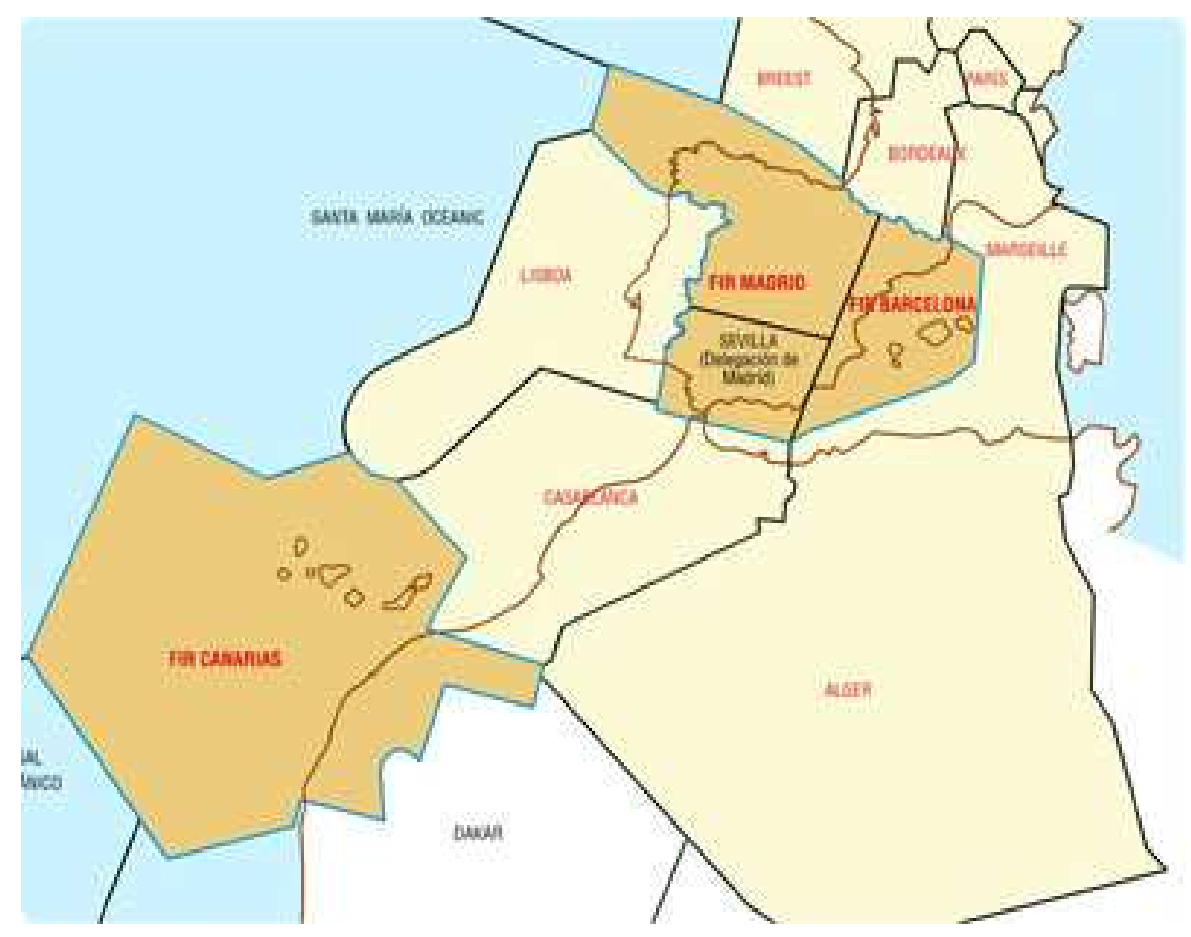

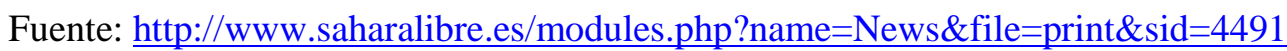

Pues bien, el documento no menciona esta circunstancia ni tampoco hace valoración alguna de las implicaciones que tales compromisos puedan tener en términos de nuestra seguridad marítima.

Por otra parte, no se establece una vinculación apropiada con otras cuestiones como el terrorismo, que en algunos casos aparecen en el texto, pero no se relacionan con la seguridad marítima de la forma en que sería aconsejable.

Al respecto cabe mencionar, no obstante, que la Estrategia de Seguridad Marítima Nacional sí analiza con profusión la relación entre la protección del espacio marítimo y la protección frente a otras amenazas y riesgos para la seguridad ${ }^{13}$.

Del mismo modo es innegable que el texto se queda muy corto en la explicación de las implicaciones que estos desafíos tienen en el caso concreto de España. Al respecto sólo se menciona el potencial impacto en el comercio internacional de doble vía -un $85 \%$ del cual se realiza por $\operatorname{mar}^{14}$ - y el abastecimiento energético, cuestiones ciertamente muy importantes, pero nada se dice de esas otras cuestiones asociadas a la vulnerabilidad del espacio marítimo y ya mencionadas más arriba.

Por otra parte, tampoco las cuestiones relacionadas con el abastecimiento energético y el comercio se abordan con el detalle que sería aconsejable en un contexto internacional cada vez más marcado por la competencia por recursos energéticos y materias primas, en el que los Estados no dudan en emplear todos los medios a su alcance para tratar de asegurar el acceso y

\footnotetext{
13 "Estrategia de Seguridad Marítima Nacional" (2013), pp. 21 y ss.

14 Del Pozo, Fernando: "La seguridad marítima hoy: la mar nunca está en calma", Real Instituto Elcano, Documento de Trabajo 3/2014 (12 de marzo de 2014), p. 3.
} 
control sobre tales recursos ${ }^{15}$. Algo doblemente sorprendente si se tiene en cuenta que España ocupa el cuarto lugar en la UE por volumen de bienes transportados por vía marítima y que puertos como Valencia o Algeciras se encuentra entre los 50 primeros del mundo si atendemos a su tráfico ${ }^{16}$.

Una falta de atención doblemente grave si se tiene en cuenta no sólo la responsabilidad de España en la protección de sus propias líneas de abastecimiento y sus rutas de paso, sino también la contribución que debe hacer en la protección de las rutas claves para los intereses de la Unión Europea en su conjunto ${ }^{17}$.

En cualquier caso, es preciso insistir en que sí se abordan con más detalle estas cuestiones en la Estrategia de Seguridad Marítima Nacional.

Por lo demás, tampoco recoge el texto de la estrategia el desafío a la libre navegación que pueden suponer los sistemas de denegación de acceso de los que disponen un número creciente de Estados y que, no sólo pueden suponer un límite a la actuación de nuestras fuerzas armadas y las de nuestros aliados en zonas en que hasta ahora habían podido desplegarse sin problema, sino que podrían servir para impedir la libre circulación de pasajeros y mercancías, incluidas materias primas ${ }^{18}$.

Sin embargo, la estrategia española no establece como prioritaria la garantía de dicha libertad de tráfico y tampoco lo hace respecto de áreas de especial trascendencia que, por ello, deberían ser objeto de especial atención y protección, como los estrechos, incluido el estrecho de Gibraltar ${ }^{19}$-uno de los principales estrechos del mundo y por el que pasan anualmente más de 100.000 buques $^{20}$ - ni respecto de áreas como el espacio entre las Islas Canarias y la península o el espacio interinsular en Canarias, también de gran importancia para España ${ }^{21}$.

En definitiva, en lo que hace a la seguridad marítima, la estrategia de seguridad nacional no contribuye como debería a identificar los riesgos para la seguridad y, consecuentemente, difícilmente podrá ser un elemento efectivo de cara a su reducción, algo para lo que también debería servir ${ }^{22}$.

\section{Líneas de acción planteadas}

\footnotetext{
15 Informe Elcano, "Hacia...", pp. 12 y 24.

${ }^{16}$ Del Pozo, op. cit., pp. 2 y ss.

17 "Towards a European Global Strategy: Securing European Influence in a Changing World", Istituto Affari Internationali; The Polish Institute of International Affairs; Real Instituto Elcano y The Swedish Institute of International Affairs (28 de mayo de 2013), pp. 12.

18 Arteaga, op. cit., pp. 14.

${ }^{19}$ Una omisión que sorprende especialmente si tenemos en cuenta que entre los ejercicios programados por la Armada Española se incluyen maniobras orientadas a la mejora de las capacidades de vigilancia y protección de buques en el Estrecho de Gibraltar; "La Armada está poniendo a prueba la seguridad de los puertos españoles", El Confidencial Digital, 15 de mayo de 2014, en

http://www.elconfidencialdigital.com/defensa/Armada-poniendo-seguridad-puertosespanoles_0_2271372856.html.

${ }^{20}$ Del Pozo, op. cit., p. 3.

21 "Hacia una estrategia de seguridad nacional para España", Centro Superior de Estudios de la Defensa Nacional, Ministerio de Defensa, Documentos de Seguridad y Defensa, no 25 (Febrero de 2009), pp. 49 y ss.

${ }^{22}$ En relación con estas funciones de la Estrategia de Seguridad Nacional ver: "Hacia una estrategia...", op. cit., pp. 16.
} 
El texto se limita a señalar que "España debe garantizar la protección de la vida humana en el mar y la seguridad de la navegación..." (p 36 y 37), pero no se explicitan claramente las líneas a seguir para ello más allá de una mejor coordinación de las administraciones públicas y un mejor empleo de los medios existentes al respecto.

En el mejor de los casos, la voluntad de intensificar la cooperación con otros Estados mediante organizaciones multilaterales y acuerdos bilaterales, la decisión de impulsar el intercambio de información y la realización de actividades conjuntas (aunque tampoco se dice cuáles ni de qué tipo) y el compromiso con el fomento de la colaboración con el sector privado ( $\mathrm{p}$ 50) cuestión en la que profundiza la Estrategia Marítima de Seguridad Nacional ${ }^{23}$ son las aportaciones más concretas y más susceptibles de tener efectos reales, cuestiones en todo caso importantes como recordaba el Real Instituto Elcano en un informe reciente ${ }^{24}$.

Al respecto, hay que decir que, precisamente en relación con un ámbito como el de la seguridad marítima, para el que las actividades delictivas de carácter transnacional suponen un importante desafío, la falta de cooperación, la inexistencia de mecanismos de gobernanza global $^{25}$ y la tendencia a la renacionalización de los planteamientos de seguridad observable en el momento actual, es particularmente preocupante.

En este sentido, parece especialmente necesario que en el marco de la Unión Europea se establezcan planteamientos sólidos de conjunto que permitan fortalecer la seguridad marítima, en línea con lo planteado por la estrategia de seguridad marítima presentada el pasado mes de marzo $^{26}$. Una estrategia que es consecuencia precisamente de la propuesta formulada en ese sentido en el Consejo Informal de Ministros de Defensa celebrado en Mallorca en febrero de 2010, bajo presidencia española de la $U^{27}$. En ese mismo sentido, parece lógico que España, como Estado eminentemente marítimo, participe activamente en su desarrollo y aplicación.

Asimismo, sería deseable determinar con precisión la contribución que puede hacer España en el marco de la UE en el contexto de los esfuerzos de ésta para la potenciación de la seguridad marítima, entre los que cabe mencionar el concepto de "operaciones de seguridad marítima de la UE”, aprobado en abril de $2012^{28}$.

Al propio tiempo será preciso mantener la cooperación en el marco de la OTAN, de la mano de instrumentos como la Estrategia Marítima de la OTAN, aprobada en 2011.

Por lo demás, tampoco se plantea adecuadamente de qué modo pueden contribuir las fuerzas armadas y cuerpos de seguridad en este ámbito ni qué tipos de capacidades serán necesarios para hacer frente a los desafíos vinculados a la seguridad marítima ${ }^{29}$, algo que resulta especialmente sorprendente porque es precisamente la Estrategia de Seguridad

\footnotetext{
23 "Estrategia de Seguridad Marítima Nacional" (2013), pp. 33 y ss.

${ }^{24}$ Informe Elcano, "Hacia...", op. cit., pp. 25.

25 Íbid., pp 12.

${ }^{26}$ Joint Communication to the European Parliament and the Council: "For an open and secure global maritime domain: elements for a European maritime security strategy", JOIN (2014) 9 final, Bruselas (6 de marzo de 2014); ver también: "La UE respalda una estrategia de seguridad marítima contra la piratería en el golfo de Guinea", La Voz de Galicia, 18 de marzo de 2014, en

http://www.lavozdegalicia.es/noticia/internacional/2014/03/18/ue-respalda-estrategia-seguridad-maritimacontra-pirateria-golfo-guinea/0003 201403G18P41995.htm.

${ }^{27}$ Molina Schmid, Teresa (2013): "Los asuntos marítimos en el marco de la Unión Europea. Una estrategia de seguridad marítima en la UE", en VV.AA.: Enfoque integral de la seguridad en el espacio marítimo español, Monografías 135, Escuela de Altos Estudios de la Defensa, Ministerio de Defensa, Agosto de 2013, p. 88.

${ }^{28}$ Íbid., p 81

29 "Estrategia de Seguridad Marítima Nacional" (2013), pp. 11.
} 
Nacional el documento que debería inspirar acciones como los ejercicios Maresc 2014, que desarrollará la Armada Española para probar y mejorar su capacidad de respuesta a posibles incidentes y problemas de seguridad marítima ${ }^{30}$.

Junto a todo ello, tampoco deja constancia el texto de la necesidad de partir de planteamientos más amplios, tanto a escala europea ${ }^{31}$ como española, que garanticen la coherencia entre las acciones puestas en marcha en materia de seguridad marítima y las acciones desarrolladas en otros ámbitos, en particular, en el caso español y como señalábamos más arriba, el ámbito de la inmigración ilegal.

\section{Conclusiones}

La lectura de la Estrategia de Seguridad Nacional, a diferencia de lo que ocurre con los principales documentos estratégicos de los Estados de nuestro entorno, pone de manifiesto que la seguridad marítima no se considera en ningún caso entre las prioridades estratégicas de nuestro país.

Tal planteamiento es incomprensible teniendo en cuenta no sólo el carácter eminentemente marítimo de España sino el hecho de que buena parte de su comercio se realiza por vía marítima.

Asimismo, otros de los principales desafíos a la seguridad española como el terrorismo, la piratería, los tráficos o la inmigración ilegal se encuentran inextricablemente unidos a la seguridad marítima, como lo es también la protección de las prospecciones marítimas en la zona económica exclusiva.

Así, el documento no profundiza en la importancia para España de garantizar la seguridad marítima ni, en consecuencia, da cuenta de la conveniencia de una adecuada implicación de España en la protección de las rutas marítimas. Del mismo modo, tampoco hace un análisis exhaustivo de las áreas geográficas que, con vistas a la protección de la seguridad marítima deben ser de atención prioritaria, pues menciona el Golfo de Guinea, pero pasa por alto otras áreas como la costa oriental africana y el Océano Índico, no menos importantes.

Junto a todo ello, se echa en falta una descripción detallada y precisa de las medidas a poner en marcha con vista a reforzar la seguridad marítima así como de las instituciones y estructuras que serán responsables de la puesta en práctica y desarrollo de tales medidas.

\footnotetext{
30 "La Armada está poniendo a prueba...", op. cit.

${ }^{31}$ En el ámbito europeo, ya en 2007 la Comisión lanzó el "Libro azul de la política marítima integrada de la Unión Europea" orientado a lograr una aproximación intersectorial a las cuestiones marítimas en el ámbito de la Unión, algo necesario ya que una decena de direcciones generales y varias agencias tienen competencias en cuestiones de carácter marítimo: ver Molina Schmid, op. cit., p 71; ver también Del Pozo, op. cit., p 17.
} 